\title{
Wilms tumor suppressor gene (WT1) suppresses apoptosis by transcriptionally downregulating $B A X$ expression in immature rat granulosa cells
}

\author{
Minji Park, Yuri Choi, Hyeonhae Choi and Jaesook Roh ${ }^{*}$
}

\begin{abstract}
Background: The important role of WT1 in early folliculogenesis was evident from its restricted expression pattern in immature follicles and from its involvement in transcriptional control of inhibin- $a$ and FSH receptor. There is also considerable evidence that WT1 is a potent inhibitor of apoptotic cell death in the developing kidney and male germ cells, suggesting that it could play a role in the regulation of follicle survival. Therefore, we evaluated if WT1 involves in cell survival of granulosa cells (GCs) during the FSH-independent stage.

Methods: GCs were obtained from small preantral follicles of immature rat ovary. Bax and bcl-2 mRNA and protein levels in GCs transfected with WT1 (-KTS) or WT1 (+KTS) were analyzed by Real-time RT-PCR and immune-blotting analysis. Cell viability was measured with MTT assays and apoptosis was analyzed with caspase 3/7 activity and TUNEL assay. The mechanism by which WT1 regulates Bax expression was investigated using Bax promoter-luciferase reporter assay and ChIP assays from GCs.

Results: Here, we showed that WT1 (-KTS) suppressed endogenous Bax transcript and protein expression, and this inhibition resulted from direct binding of WT1 in the Bax promoter region in vivo. In addition, anti-apoptotic effects of WT1 (-KTS) were demonstrated based on MTT assays, a sensitive bioluminescence caspase 3/7 assay and TUNEL assays. On the other hand, WT1 has no role on bcl-2 expression in GCs.

Conclusion: These findings suggest that activation of WT1 is necessary for maintenance of GC survival during early stage of follicles and WT1 can play a role in protecting apoptosis through the regulation of upstream activator (Bax), as well as through regulation of downstream effecter (caspases 3 and 7).
\end{abstract}

Keywords: WT1, Apoptosis, Bax, bcl-2, Caspase, Granulosa cell, Rat

\section{Introduction}

The fate of the follicles depends on the balance between anti- and pro-apoptotic factors that determine the rate of granulosa cell (GC) apoptosis. Although apoptosis can occur at any stage of follicular development, a growing follicle is more prone to apoptosis than arrested or early-stage follicles, and the majority of follicles become atretic during the antral stage [1]. The factors regulating follicular growth and survival appear to differ for small follicles and the more differentiated antral follicles. Further study of how the apoptotic genes are regulated may

\footnotetext{
* Correspondence: rohjaesook@hanyang.ac.kr

Laboratory of Reproductive Endocrinology, Department of Anatomy and Cell Biology, College of Medicine, Hanyang University, San17 Haengdang-dong, Seongdong-gu, Seoul 133-791, South Korea
}

answer the questions of why quiescent follicles are not apoptotic, and why early-stage follicles are relatively resistant to apoptosis until they receive appropriate signals to initiate further growth.

The important role of $W T 1$ in early folliculogenesis is evident from the fact that its expression is restricted to immature follicles [2] and from its involvement in the transcriptional control of ovarian marker genes that encode inhibin- $\alpha$ [3] and the follicle stimulating hormone (FSH) receptor [4]. There is also considerable evidence that WT1 is a potent inhibitor of apoptotic cell death in the developing kidney [5] and male germ cells [6], suggesting that it could play a role in the regulation of follicle survival. However, there is no direct evidence of an anti- or pro-apoptotic function of WT1 protein, and its 
precise mechanism of action in the ovary is not well understood.

We hypothesized that WT1 was required to regulate the transcription of the genes that provide a cell survival advantage to the GCs of the early preantral follicles in the FSH-independent stages of development. Here we investigated whether the expression of WT1 was associated with changes in the expression of two apoptosis related genes, $b c l-2$ and $B a x$, which function as the final common mediators of life and death [7]. In addition, we examined the effects of WT1 on GC viability and apoptosis. A better understanding of the cellular signals that induce or prevent apoptosis may help us control follicular development and rescue more oocytes from quiescent early follicles.

\section{Materials and methods}

Animals

Immature female Sprague Dawley rats were obtained from Samtako Biokorea (Kyunggi, South Korea). All animals were housed under controlled humidity, temperature, and light conditions, and fed standard rat chow ad libitum. Animal care was consistent with institutional guidelines, and the Hanyang University ACUC committee approved all procedures involving animals (HY-IACUC-09-034).

\section{Preparation and culture of granulosa cells}

GCs were obtained by puncture of the ovaries of immature 25-day-old rats (body weight, 5565 g) previously primed with diethylstilbestrol. The ovaries were punctured in Leibovitz L-15 medium. Ovarian debris was removed, and the remaining medium containing GCs was collected after low-speed centrifugation at $500 \mathrm{~g}$ for $10 \mathrm{~min}$. The GCs were dispersed by repeated washing and suspended in growth medium (McCoys 5a supplemented with $2 \mathrm{mM}$ L-glutamine, $100 \mathrm{U} / \mathrm{mL}$ penicillin, and $100 \mu \mathrm{g} / \mathrm{mL}$ streptomycin). McCoys 5a medium (modified) and Leibovitz L-15 medium were obtained from GIBCO (Santa Clara, CA, USA). Penicillin and streptomycin were obtained from Sigma (St. Louis, MO, USA). Recombinant human FSH (Org 32489E) was from NV Organon (Oss, The Netherlands).

\section{Plasmid construction and transfection}

Full-length mouse WT1 cDNA (-KTS) or (+KTS) was subcloned into the pCMV5 expression vector under the control of the CMV promoter and the GH-pA signal, [WT1 (-KTS)] or [WT1 (+KTS)]. Granulosa cells (5 $10^{5}$ viable cells/well) were grown in culture medium supplemented with $10 \%$ fetal bovine serum (FBS) for $2 \mathrm{~h}$. The medium was then changed to serum-free medium and the cells were transfected with expression and/or reporter plasmids using Lipofectamine 2000 reagent (Invitrogen, Carlsbad, CA, USA) according to the manufacturers instructions. The cells were used for the experiment at sixteen hours after transfection. At the end of growth they were frozen for RNA or protein extraction.

To evaluate promoter activity induced by WT1 overexpression, cells were cotransfected with the $\operatorname{Bax}(-2673$ to +1 bp of the 5 flanking sequence of the mouse $B a x$ gene) promoter-luciferase reporter plasmid Bax-Luc (250 ng) with WT1 (-KTS), or WT1 (+KTS) cDNA (100 ng) or empty vector as a balancer. The p-Rous sarcoma virus (RSV)- $\beta$-galactosidase (gal) vector (50 ng) containing the lac $Z$ gene encoding $\beta$-gal driven by the RSV long terminal repeat was used as an internal control to correct for differences in transfection efficiency. Sixteen hours after transfection, the cells were incubated in FSH $(50 \mathrm{ng} / \mathrm{mL})$ or control medium for $1624 \mathrm{~h}$, then harvested, lysed, and assayed for luciferase activity.

To harvest the cells, lysis buffer $(200 \mu \mathrm{L})$ (Promega) was added to each well and $30 \mu \mathrm{L}$ of supernatant was used to detect luciferase activity on a Monolight 2010 luminometer (Analytical Luminescence Laboratory, San Diego, CA, USA). $50 \mu \mathrm{L}$ of cell lysate was also used to measure $\beta$-gal activity. The activity of the promoter is expressed as the ratio of relative light units $/ \beta$-gal activity.

\section{Real time RT-PCR}

Total RNA was isolated with an RNeasy extraction kit (Qiagen Inc., Valencia, CA, USA). $1 \mu \mathrm{g}$ aliquots of total RNA were annealed ( $5 \mathrm{~min}$ at $70 \mathrm{C}$ ) to oligo(dT) 18 primers and reverse transcribed using an Advantage RT-for-PCR kit (BD Biosciences; Clontech, Palo Alto, CA, USA). The primers were designed for the mRNA sequences of $\mathrm{Bax}$ and $b c l-2$ using the Primers Express program (PE Applied Biosystems, Foster City, CA, USA): Bax forward, 5 -TGT TTGCTGATGGCAACTTC-3 and reverse, 5-GATCAG CTCGGGCACTTTAG-3 (GenBank accession no. NM 017059.1); $b c l-2$ forward, 5-GGGATGCCTTTGTGGA ACTA-3 and reverse, 5 -CTCACTTGTGGCCCAGGT AT-3 (GeneBank accession no. NM_016993.1). GAPDH was amplified using 5-GCTGGCATTGCTCTCAAT GACA-3 (forward) and 5-TCCACCACCCTGTTGC TGTA-3 (reverse) (GenBank accession no. NM_017008) to normalize each reaction (amplification product sizes 104,138 , and 83 bps for $B a x, b c l-2$, and $G A P D H$, respectively). Real-time polymerase chain reactions were carried out in $25 \mu \mathrm{L}$ volumes using SYBR Green Supermix (BioRad, Hercules, CA, USA) in an iCycler Thermal Cycler (BioRad). Samples were run in triplicate in 96-well optical plates (BioRad) and mean values were compared with the control values (empty vector-transfected cells) to calculate the relative amount of transcript.

\section{Western blotting}

To investigate $B a x$ and $b c l-2$ protein regulation by $W T 1$, GCs transfected with WT1(-KTS) or WT1(+KTS) were 
harvested after $24 \mathrm{~h}$ culture and washed once on ice with cold PBS before lysis in Laemmli buffer containing $\beta$-mercaptoethanol. The cells were gently sonicated on ice for $15 \mathrm{sec}$ with an MSE sonicator (Sanyo Corp., Osaka, Japan) and boiled for $3 \mathrm{~min}$. Proteins were separated on $8 \%$ SDS-PAGE gels and electroblotted onto Hybond-P membranes (Amersham Pharmacia Biotech, Arlington Heights, IL, USA). To detect WT1, Bax, and $b c l-2$, the membranes were blocked for $1 \mathrm{~h}$ at room temperature in TBS-0.1\% Tween containing 5\% fat-free dry milk, and incubated with anti-WT1 antibody (C19; Santa Cruz Biotechnology, Inc., Santa Cruz, CA, USA), anti-Bax antibody (B-9, SC 7480), or anti-bcl-2 antibody (C-2, SC 7382) diluted 1:500 for $3 \mathrm{~h}$ at room temperature. The membranes were washed and blotted with peroxidase-conjugated donkey anti-rabbit secondary antibody $(1: 8,000)$ (Boehringer Mannheim, Indianapolis, IN, USA) for WT1, or with peroxidase-conjugated goat antimouse secondary antibody (1:5,000) (W402B, Promega, Madison, WI, USA) for Bax and $b c l-2$. Immunolabeled proteins were detected using an enhanced chemiluminescence kit (Amersham Pharmacia Biotech). The bands corresponding to $W T 1, B a x$, and $b c l-2$ proteins (respectively, 52,22 , and $26 \mathrm{kDa}$ ) are indicated in Figure $1 \mathrm{C}$ and $\mathrm{D}$. To ensure the cell lysates were loaded equally, the blots were stripped and incubated with $\beta$-actin (1:1,000 dilution;
Sigma, St. Louis, MO, USA). Data were collected from at least two independent experiments.

\section{Analyses of cell viability and apoptosis}

Cell viability was determined using an MTT [3-(4,5dimethythiazol-2-yl)-2,5-diphenyltetrazolium bromide] assay kit (Cayman Chemical Company, Ann Arbor, MI, USA). The conversion of MTT into the aqueous soluble formazan product is accomplished by dehydrogenase enzymes found in metabolically active cells. GCs were cultured in McCoy 5a serum-free medium with or without FSH or $10 \%$ FBS for $24 \mathrm{~h}$ after transfection. After addition of tetrazolium salt solution and incubation at $37 \mathrm{C}$ for $2 \mathrm{~h}$, the quantity of the formazan product was measured by the absorbance at $570 \mathrm{~nm}$. The experiment was repeated three times, and the data were expressed as fold changes relative to the control values measured with empty vector-transfected cells cultured for $24 \mathrm{~h}$ in the absence of serum.

Apoptosis was subsequently assessed with the caspase $3 / 7$ activity assay using a luminescence assay kit (CaspaseGlo 3/7 assay; Promega). GCs were cultured in McCoy 5a serum-free medium with or without FSH or 10\% FBS for $24 \mathrm{~h}$ after transfection. Then proluminescent Caspase-Glo $3 / 7$ reagent was added to each well, and the cells were gently mixed and incubated at room temperature for $1 \mathrm{~h}$.
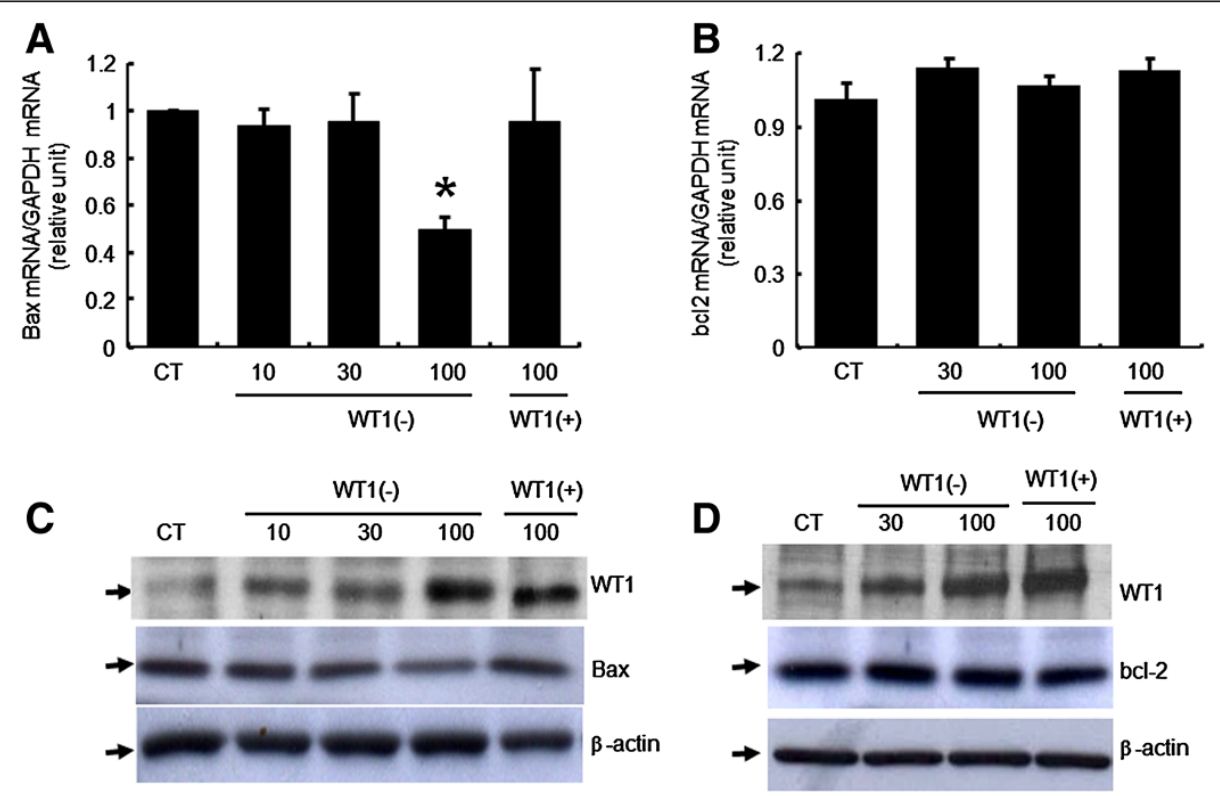

Figure 1 Regulation of Bax and bcl-2 expression by $W T 1$ in immature rat GCs. Real-time RT-PCR analysis of (A) Bax and (B) bcl-2 mRNA levels in GCs transfected with increasing amounts of WT1 (-KTS) (10, 30, 100 ng/well) or WT1(+KTS) (100 ng/well). GAPDH was used to normalize each reaction. Values are calculated as fold changes relative to control (CT), and are expressed as the mean SD of three independent experiments, each performed in triplicate. $C T$, cells transfected with empty vector. ${ }^{*} p<0.05$ compared to control. Western blot analysis of (C) Bax and (D) bcl-2 protein levels. Lysates were immunoblotted with anti-WT1 antibody (C-19; Santa Cruz Biotechnology, Inc.), anti-Bax antibody (B-9, SC 7480), or anti-bcl-2 antibody (C-2, SC 7382). Arrows indicate bands corresponding to WT1 (52 kDa), Bax (22 kDa), bcl-2 (26 kDa) and B-actin (42 kDa), respectively. 
Luminescence was measured on a Monolight 2010 luminometer (Analytical Luminescence Laboratory, San Diego, CA, USA). As for the MTT assay, the data were expressed as fold changes relative to the control.

\section{Terminal deoxynucleotidyl transferase-mediated dUTP-biotin nick end labeling (TUNEL) assay}

Apoptosis was analyzed by 3 -end labeling of DNA fragments in situ using the ApopTag In-Situ Apoptosis Detection kit (Oncor, Gaithersberg, MD) according to the manufacturer s protocol. In addition, we performed fluorescent staining for WT1 protein to confirm overexpression of WT1 in transfected GCs. GCs ( $110^{5}$ cells) were plated onto $22 \mathrm{~cm}$ coverslips at 6-well plate and transfected with WT1 or empty-vector for $6 \mathrm{~h}$ and then serum starved for another $36 \sim 48 \mathrm{~h}$. After then, cells were fixed with $4 \%$ paraformaldehyde for $10 \mathrm{~min}$ at room temperature and washed twice in PBS. The cells were then incubated with terminal deoxynucleotidyl transferase and digoxigenin-11 dUTP in a humidified chamber at $37 \mathrm{C}$ for $1 \mathrm{~h}$ or polyclonal anti- WT1 antibody (1:100) for $2 \mathrm{~h}$ at room temperature. The cells were washed with PBS and incubated with antidigoxigeninfluorescein antibody for $1 \mathrm{~h}$ for TUNEL or a Alexa Flour 594 goat antirabbit IgG (1:200)(Invitrogen, Carlsbad, CA, USA) for $30 \mathrm{~min}$ for WT1. The cells were then washed with buffer and nuclear DNA was counterstained with 4,6-diamidino-2-phenylindole (DAPI)(Vector Laboratories, Inc., Burlingame, CA, USA). We counted the number of apoptotic cells using a Leica TCP SP5 confocal microscope (Leica, Heidelberg, Germany). We randomly selected $4 \sim 6$ magnified fields (X200) and calculated the average number of apoptotic cells per field. Apoptosis was expressed as the percentage of TUNEL-positive nuclei per field. These experiments were repeated five times.

\section{Chromatin immunoprecipitation (ChIP) analysis}

ChIP assay was performed on WT1 in the Bax promoter region using a ChIP kit (Upstate Biotechnology, Inc., Lake Placid, NY) according to the manufacturer s protocol with minor modifications. Briefly, immature rat GCs $\left(210^{7}\right)$ were transfected with WT1(-KTS) using lipofectamine 2000, cultured in McCoy 5a serum-free medium for $24 \mathrm{~h}$, and cross-linked with $1 \%$ formaldehyde for $10 \mathrm{~min}$ at room temperature. The reaction was terminated by the addition of glycine (final concentration, $0.125 \mathrm{M}$ ) for $5 \mathrm{~min}$ at room temperature. Cells were pelleted by centrifugation and lysed in $1 \mathrm{ml}$ ice-cold lysis buffer containing protease inhibitor cocktail (Roche Applied Science, Indianapolis, IN, USA). The lysates were sonicated on ice at a $250 \mathrm{~W}$ power level for 25 min with 15-sec sonication and 30-sec intervals with a Bioruptor KR (CosmoBio Co., Ltd., Tokyo, Japan) to obtain DNA fragments of an average length of approximately 100 500 bp. Chromatin was immunoprecipitated overnight at 4C with anti- WT1 antibody (10 ug/reactions; Santa Cruz Biotechnology, Inc., Santa Cruz, CA, USA) or normal rabbit IgG (5 ug/reaction; Santa Cruz Biotechnology, Inc., Santa Cruz, CA, USA) as a negative control. Immune complexes were collected using protein G-agarose slurry for $2 \mathrm{~h}$ at $4 \mathrm{C}$ with rotation and sequentially washed for $3 \mathrm{~min}$ each in low salt wash buffer, high salt wash buffer, $\mathrm{LiCl}$ wash buffer, and two washes with Tris/ EDTA buffer. Precipitates were then extracted two times with elution buffer. Eluates were pooled, and crosslinking was reversed by incubation at $65 \mathrm{C}$ overnight. Unbound proteins were digested with proteinase $\mathrm{K}$ (Promega) for $2 \mathrm{~h}$ at $45 \mathrm{C}$, and chromatin was purified using the DNA Clean-up kit (GeneAll Biotechnology, Seoul, Korea). DNA was analyzed by PCR using the primers designed to amplify fragments of the WT1 motif in the Bax promoter [see Figure $2 \mathrm{~A}, \mathrm{WT} 1^{-1497}$ (forward 5 GGC CTG CTG CTA CTT CAC AT-3, reverse 5-TTT TAA TCC CAG CCC TCA GA-3), WT1 ${ }^{-1299}$ (forward 5 TAG TCC AGG CTG ACC TCG AA-3, reverse 5-ATG GTG GCC CAT ACC TGT AA-3), WT1 ${ }^{-1160}$ (forward 5 CAT CCT AGG CTG GCT TCA GA-3, reverse 5 -CCT TCT CAC CTC ACC ATA CCT C-3), WT1 ${ }^{-1008}$ (forward 5 -GAG GTA TGG TGA GGT GAG AAG G-3, reverse 5 TCT CTC TCC TCC TTT CCC AAA-3), and WT1 ${ }^{-239}$ (forward 5-ATA TCC CAG GCA AGC TTT GA-3, reverse 5-GCC GCG GGT ACT AAA TGA AC-3)]. After 25 - to 28-cycle amplification, PCR products were run on a $1.5 \%$ agarose gel, stained with ethidium bromide, and visualized under UV light.

\section{Data analysis}

All results are expressed as the mean SD of duplicate measurements of triplicate cultures, and each experiment was repeated at least three times. Statistical significance was determined by Mann Whitney $U$-test for two group comparison and Kruskal-Wallis one-way analysis of variance for multiple group comparisons. Significance was accepted at $\mathrm{p}<0.05$.

\section{Results}

\section{Effects of WT1 overexpression on basal Bax and bcl-2 transcript and protein levels}

To determine whether WT1 regulates the endogenous expression of pro-apoptotic genes directly, we analyzed primary GCs expressing either WT1 (+KTS) or WT1 (-KTS). Since insertion or removal of the three amino acid sequence (KTS) changes the DNA-binding specificity of WT1 $[8,9]$, it was important to compare the effects of the two isoforms in GCs. Markers of apoptosis (i.e. Bax or $b c l$-2) were detected using real time RT-PCR and Western blotting. Overexpression of WT1 (+KTS) 

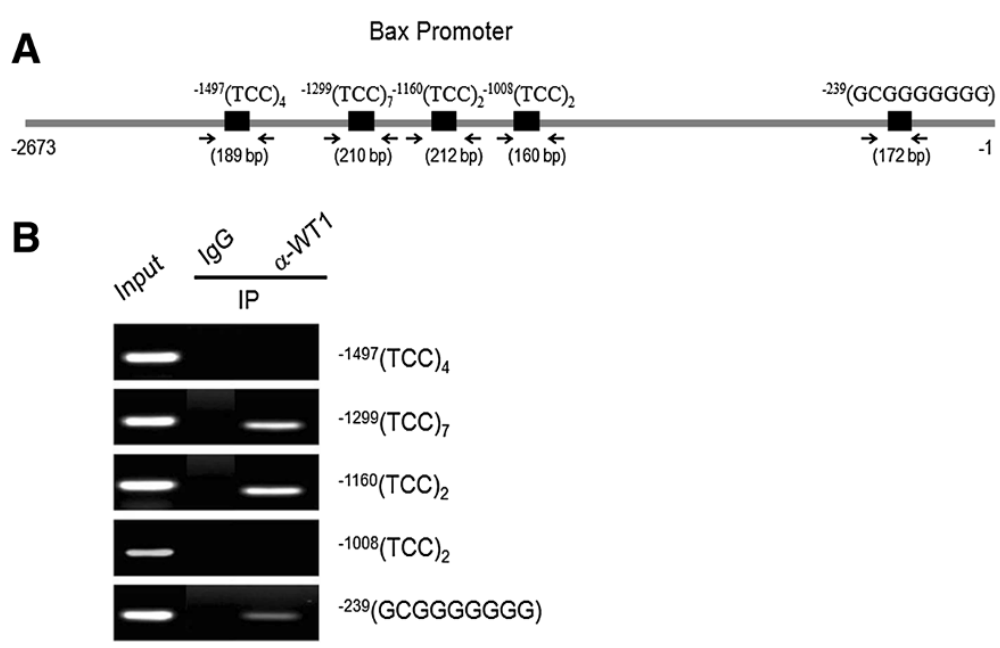

Figure 2 Evidence of WT1 binding in the Bax promoter region in vivo. (A) Schematic showing the Bax promoter and the target region tested in ChIP assay. The locations of primers used to amplify DNA fragments spanning WT1 transcription binding sites were designated in the Bax promoter region. (B) ChIP detection of WT1 transcription factor binding to the Bax promoter region in immature GCs. ChIP assays were performed using DNA extracted from GCs obtained at $3648 \mathrm{~h}$ after transfection. One tenth of the chromatin was kept as input DNA control (Input) before immunoprecipitation. Immunoprecipitations were performed with WT1 antibody, or normal rabbit lgG served as a negative control. DNAs were analyzed by PCR using primers indicated previously. WT1 $1^{-1299}(210 \mathrm{bp})$ and WT1 ${ }^{-1160}$ (212 bp) DNA fragments containing WT1 transcription factor binding sites were enriched in chromatin samples immunoprecipitated with WT1 antibody as well as input DNA. A representative gel picture from at least four independent experiments is shown.

and WT1 (-KTS) in GCs was confirmed, and their corresponding immunoreactive bands $(52 \sim 54 \mathrm{kDa})$ are shown in Figure $1 C$ and D (upper lane). Total RNA was isolated from the cells expressing either the WT1 (-KTS) or the WT1 (+KTS) isoform. Interestingly, in the GCs expressing the WT1 (-KTS) isoform, we observed significantly decreased levels of $\operatorname{Bax}$ transcripts, while there was no significant effect in the GCs expressing WT1 (+KTS) (Figure 1A). It was important to determine whether this decrease led to a subsequent decline in the levels of Bax protein. To address this question, total proteins were isolated from GCs expressing either WT1 (+KTS) or WT1 (-KTS) proteins. Consistent with the findings of real time RT-PCR, the cells overexpressing WT1 (-KTS) contained less Bax protein than the empty vector-transfected cells (Figure 1C), while the GCs expressing the WT1 (+KTS) isoform had the control level of Bax (Figure 1C). The levels of $b c l-2$ transcripts and protein were not altered by overexpression of either of the two WT1 isoforms (Figure 1B and D).

\section{Effect of WT1 overexpression on GC viability under serum deprivation}

Despite its tumor suppressor function, WT1 prevents programmed cell death in some cell types but promotes it in others [10-12]. Thus, we were interested to find out whether WT1 protein altered sensitivity to apoptotic stimuli. To investigate the role of WT1 in GC viability, we employed the MTT assay to monitor the activity of mitochondrial reductase [13]. GCs transfected with WT1 were cultured with or without serum or FSH for $24 \mathrm{~h}$. The purpose of removing the serum was to induce spontaneous onset of GC apoptosis because serum has shown to contain growth factors associated with a significant decrease in pro-apoptotic proteins [14]. On the other hand, FSH is the main physiological regulators of ovarian follicle survival and sufficient FSH concentrations are critical for survival of follicles $[15,16]$.

As shown in Figure 3A, the effect of WT1 (-KTS) overexpression on MTT activity was significant $(\mathrm{p}=0.029$ compared to the control), and as potent as that of $10 \%$ FBS. In addition, we confirmed the role of FSH and serum as survival factors in immature GCs; addition of serum had a more potent effect than FSH, which may be related to the lower numbers of FSH receptors in immature GCs. Overexpression of WT1 (+KTS) also increased MTT activity, but the effect was not statistically significant ( $p>0.05$ ).

\section{Effects of WT1 overexpression on apoptosis in cultured GCs}

To see whether the enhanced cell viability was due to suppression of apoptosis, we analyzed apoptosis quantitatively by measuring caspase 3/7 activity. After incubation of the transfected cells for approximately $1624 \mathrm{~h}$, GCs were cultured with or without serum or FSH. The enzymatic activities measured in each group were compared with the activities in the cells cultured in serumfree conditions (control). As shown in Figure 3B, in the 

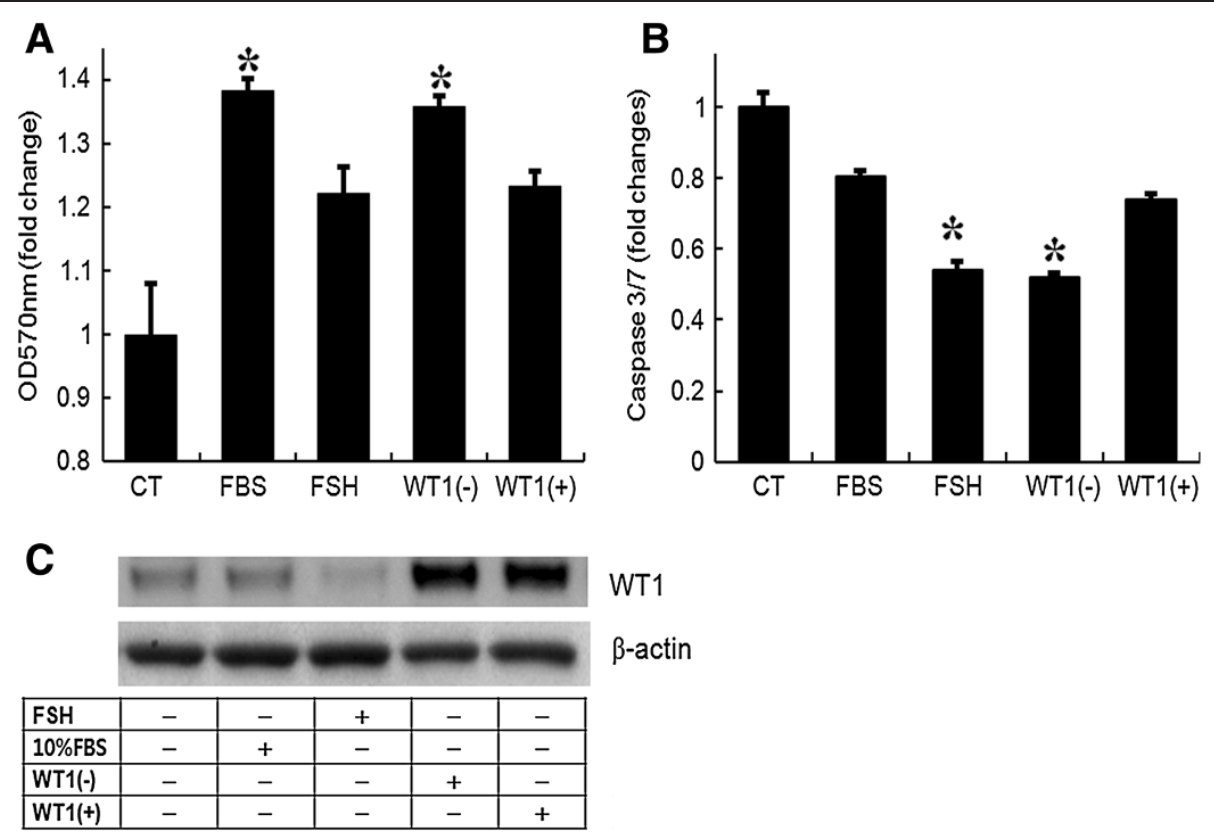

WT1

$\beta$-actin

Figure 3 Regulation of cell viability by overexpression of WT1 (-KTS), but not WT1 (+KTS). (A) MTT assays of immature rat GCs transfected with one of the two WT1 isoforms. GCs transfected with WT1 or empty vector were incubated with or without serum for $24 \mathrm{~h}$. Some cells were treated with FSH $(50 \mathrm{ng} / \mathrm{mL})$ as a positive control for survival factors. After the cells were incubated with tetrazolium salt solution for $2 \mathrm{~h}$, the quantity of formazan product was determined from the absorbance at $570 \mathrm{~nm}$. Each bar represents the fold change compared to control (CT). (B) analysis of caspase 3/7 activity. GCs transfected with WT1 or empty vector were incubated with or without serum. Some cells were treated with FSH (50 ng/mL) as a positive control for apoptosis suppressors. 24 h later, caspase 3/7 activity was measured with the bioluminescence assay. (C) Immunoblot analysis of WT1 protein in cultured GCs lysates to confirm WT1 overexpression. Bands corresponding to WT1 (52 kDa), and $\beta$-actin (42 kDa), respectively. CT, empty vector-transfected cells cultured without serum for 24 h. WT1 (-KTS) (100 ng/well); WT1 (+KTS) (100 ng/well); FBS, $10 \%$ fetal bovine serum. Data are expressed as the mean SD of three separate experiments. ${ }^{*} p<0.05$ compared to control.

GCs cultured with $10 \%$ FBS, caspase 3/7 activity was 0.8 -fold of the activity in cells cultured in serum-free conditions (control) ( $\mathrm{p}>0.05)$. Interestingly, overexpression of WT1 (-KTS) caused an approximately 0.5 -fold decrease in caspase-3/7 activity compared to the cells cultured in serum-free conditions $(\mathrm{p}=0.0026)$, indicating that WT1 (-KTS) had an inhibitory effect on GC apoptosis induced by serum deprivation. Moreover, this inhibitory effect was as potent as that of FSH. WT1 (+KTS) overexpression also decreased caspase 3/7 activity as much as $10 \%$ FBS, although the resulting activity was not significantly different from the control $(p>0.05)$. In summary, the expression of WT1 (-KTS) in GCs seems to confer significant resistance to apoptotic cell death induced by serum deprivation, an effect as potent as FSH treatment.

In addition, the effects of WT1(-KTS) on the incidence of GC apoptosis were determined by TUNEL staining in GCs (Figure 4). This was carried out in transfected GCs induced to undergo apoptosis in serum-free cultures [14]. WT1 protein expression was profoundly increased in WT1 transfected GCs and confirmed efficient transfection (Figure 4A). The percentage of apoptotic cells significantly decreased at $3648 \mathrm{~h}$ of culture in WT1 transfected cells as compared with the control (CT vs. WT1; $41.915 .7 \%$ vs. 19.7 10.3\%) $(\mathrm{P}<0.01)$

(Figure 4B). These results showed that apoptosis was suppressed in GCs transfected with WT1 compared with the control, suggesting that WT1 is involved in GCs survival by regulating apoptosis.

\section{Role of WT1 (-KTS) in suppressing Bax promoter activity}

The presence of potential binding elements for WT1 in the Bax promoter suggested that WT1 might affect Bax expression in the ovary (Figure 2A). Transient transfection assays were performed to determine whether the decrease in Bax levels, observed when WT1 (-KTS) was overexpressed, was due to the ability of WT1 (-KTS) to reduce $B a x$ promoter activity. For comparing luciferase activities, cells transfected only with the Bax promoter construct, without any treatment, constituted the control group. Increased level of WT1 protein expression in WT1 transfected GCs was confirmed (Figure 5B). As shown in Figure 5A, the WT1 (-KTS) transfected cells displayed a significantly reduced level of $\mathrm{Bax}$ promoter activity (0.6-fold of the control group) ( $<<0.05)$. As expected, FSH significantly suppressed Bax promoter activity (0.5-fold of the control) and the addition of FSH to 

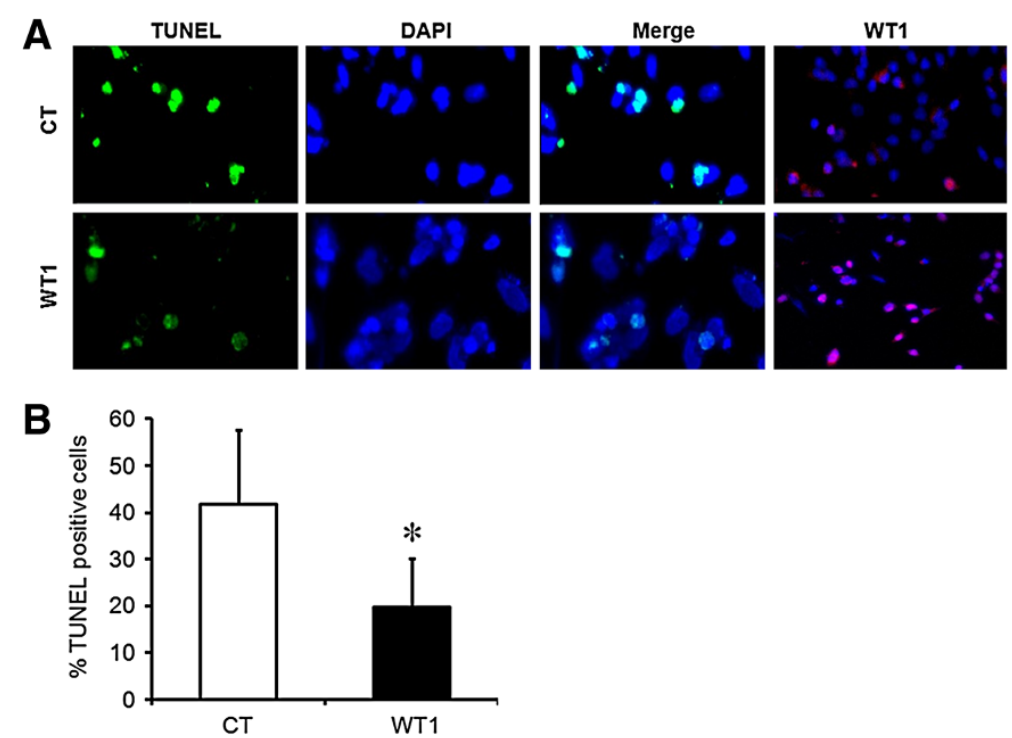

Figure 4 WT1 suppresses granulosa cell apoptosis. (A) GCS transfected with WT1(-KTS) or empty vector were stained with TUNEL or WT1 antibody to visualize apoptotic cells (green) or WT1 protein (pink: merge with DAPI) and counterstained with DAPI to confirm nuclear status (blue). Representative fluorescence confocal microscopy images are shown. Magnification; X100 or X200. (B) Frequency of TUNEL-positive nuclei in GCS transfected with empty vector or WT1(-KTS). Data represent mean SD of five independent experiments. CT, empty vector-transfected cells; WT1, GCs transfected with WT1 (-KTS)(100 ng/well). * $p<0.05$ vs. EV.
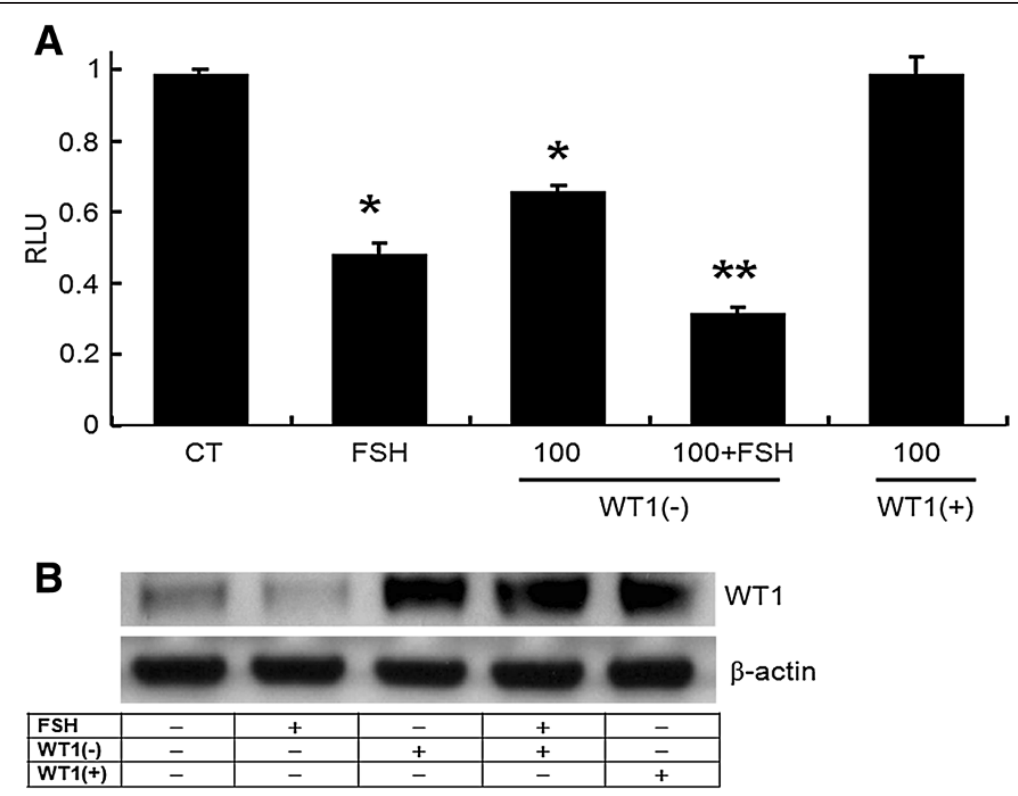

Figure 5 WT1 (-KTS) suppression of Bax promoter activity in immature rat GCs. (A) Cells were co-transfected with the Bax promoter luciferase reporter construct (250 ng/well) and either WT1 (+KTS) or (-KTS) (100 ng/well), and cultured for $24 \mathrm{~h}$. FSH (50 ng/mL) was used as a positive control for Bax repressor. Cell lysates were assayed for the activity of the luciferase reporter gene. Luciferase activity is expressed as relative light units (RLU) and normalized based on $\beta$-gal activity in co-transfected cells. Values are calculated as fold-changes relative to control (CT), and are expressed as the mean SD of three independent experiments, each performed in triplicate. (B) Immunoblot analysis of WT1 protein in cultured GCs lysates to confirm WT1 overexpression. Bands corresponding to WT1 (52 kDa), and $\beta$-actin (42 kDa), respectively. CT, control (cells transfected with Bax promoter only). ${ }^{*} p<0.05$ compared to control; ${ }^{* *} p<0.05$ compared to FSH. 
WT1 (-KTS) transfected cells further inhibited Bax promoter activity $(0.3$-fold of the control) $(\mathrm{p}<0.01)$. Conversely, WT1 (+KTS) failed to affect Bax promoter activity. Collectively, these results provide evidence that the Bax promoter is transcriptionally down-regulated by the WT1 (-KTS) isoform but not by the WT1 (+KTS) isoform.

\section{Identification of the WT1(-KTS) binding sites on the Bax promoter region in GCs}

To determine whether WT1 directly regulates Bax expression, we first analyzed the putative promoter region [ 2.7-kb upstream of transcription start site] of the Bax gene using a web-based transcription factor prediction program. This analysis revealed the presence of several putative WT1 binding sites, with five consensus binding sites (Figure 2A). To examine whether WT1 specifically binds to these candidate sites in the $\mathrm{Bax}$ promoter in vivo, ChIP assays were performed using immature GCs. PCR fragments [210 bp $(-1394 /-1185), 212$ bp $(-1253 /-1042)$ and 172 bp $(-331 /-160)]$ containing the WT1 binding sequence $\left[\mathrm{WT} 1^{-1299}(\mathrm{TCC})_{7}, \mathrm{WT}^{-1160}\right.$ $(\mathrm{TCC})_{2}$ and WT1 ${ }^{-239}$ (GCGGGGGGG), respectively] in the proximal promoter region were enriched in chromatin samples treated with WT1 antibody compared with normal rabbit IgG, demonstrating the in vivo binding of WT1 on the TCC repeats of the middle region of Bax promoter $\left[{ }^{-1160}(\mathrm{TCC})_{2},{ }^{-1299}(\mathrm{TCC})_{7}\right]$ and the GNGGGGGGG in the proximal (Figure 2B), whereas no PCR fragment was amplified in the proximal and distal $(\mathrm{TCC})_{\mathrm{n}}$ region $\left[{ }^{-1008}(\mathrm{TCC})_{2},{ }^{-1497}(\mathrm{TCC})_{4}\right]$.

\section{Discussion}

The apoptotic death of GCs underlies the initiation and progression of follicular atresia [17]. A number of factors are thought to regulate the apoptosis of ovarian GCs [18], but relatively little is known about the survival of preantral follicles compared with the later stages of development. Although responsiveness to FSH plays a critical role in the follicular transition and follicle survival in the ovary $[15,16]$, it is insufficient to prevent apoptosis during the initial, FSH-independent stages. In fact, the early antral follicles are more susceptible to apoptosis than preantral follicles $[1,19]$. Thus, our main aim was to identify how early-stage follicles remain healthy and non-apoptotic. To this end, we investigated the effects of WT1 on the expression of pro-apoptotic genes and on cell survival in immature GCs.

It is widely accepted that the relative levels of tumor suppressors, apoptotic proteins, and survival factors in GCs determine the fates of early-stage follicles [20]. WT1, a member of the zinc-finger family of transcription factors, is highly expressed in GCs in early-stage follicles. Its expression undergoes dynamic changes during folliculogenesis, alongside changes in the spatial and temporal expression of the FSH receptor gene [3]. Once the early antral follicles begin expressing FSH receptors, they become dependent on FSH stimulation for survival [1], and WT1 expression declines [2]. This stage-dependent regulation of $W T 1$ and its target genes, including the FSH receptor [4], suggests that a functional link between WT1 and FSH signaling promotes the transition of follicles from small to more differentiated. The role of FSH as the main physiological regulator of ovarian follicle cell proliferation and survival is well established [18]. Since FSH acts as a key survival factor for the growing follicles [1], the small increase in cell numbers observed after FSH treatment (Figure 3) may be the result of a low responsiveness to FSH of immature GCs obtained from small preantral follicles. As a follicle grows, expression of the FSH receptor continues to increase [21] and FSH can exert its effects on downstream molecules, including WT1 [22]. Therefore, given that WT1 enhanced GC survival in this study, it is reasonable to assume that WT1 plays a role in preventing apoptosis during the FSH-independent stages, until FSH takes on that role.

$B c l-2$ family proteins, including $b c l-2$ and $B a x$, regulate the release of pro-apoptotic factors, and in particular of cytochrome c, from the mitochondrion into the cytosol [23], which is an almost universal event during apoptotic cell death. The product of the $b c l-2$ gene prevents apoptosis, and Bax opposes bcl-2 action [24]. The relationship between $W T 1$ and the $B c l-2$ family of proteins during GC apoptosis has not been well studied. Previously, WT1 activation was shown to regulate several pro-apoptotic genes, including $\mathrm{Bax}$, in non-gonadal cells $[10,12,25]$. Bax expression was shown to be regulated differently depending on cell lineage and isoforms. For instance, $B a x$ expression was significantly decreased by either of the two WT1 isoforms in breast cancer cells [25], whereas it was not changed in leukemic cells [11]. In the cells obtained from the ovary or the uterus, the WT1 (-KTS) isoform was reported to be more potent than WT1 (+KTS) as a regulator of ovarian marker genes $[3,4]$. Likewise, our results demonstrated a suppressive role of WT1 (-KTS), but not of WT1 (+KTS), on Bax expression in GCs. A possible explanation is that the KTS insertion disrupts the critical spacing between the zinc fingers resulting in a severe reduction of binding to the consensus WT1 binding site [26]. However, a direct interaction between WT1 and the Bax promoter has not been previously demonstrated. The WT1 (-KTS) isoform binds to GC-rich sites (known as EGR1, WRE, and WTE) and to (TCC)n repeat elements [27]. An analysis of genomic sequences demonstrated the presence of potential binding elements for WT1 (-KTS) in the Bax promoter region, as shown in Figure 2A. We also showed that Bax inhibition coincided with increasing levels 
of WT1, and FSH in cells overexpressed WT1 (-KTS) further inhibited Bax promoter activity in GCs, as shown in Figure 5A. In addition, our results demonstrated that WT1 (-KTS) down-regulates endogenous Bax levels by transcriptionally regulating the $B a x$ promoter through a highaffinity WT1-binding site (Figure 2B).

On the other hand, WT1 appears to have no role in bcl-2 expression in GCs, even though it was shown to either suppress or activate $b c l-2$ in non-gonadal cells $[10,28]$. Apoptosis in follicles in vitro is mainly associated with a marked increase in Bax levels with no significant change in $b c l-2$ [29]. Since the levels of bcl-2 stay relatively constant, it is likely that the WT1enhanced cell viability is due to the markedly reduced expression of Bax (Figure 1). As WT1 is expressed in the GCs of primordial and primary follicles that are poor in FSH receptors, it is unlikely that FSH plays an important role in regulating Bax expression at this stage. This, in turn, suggests that WT1 may change the apoptotic set point by up- or down-regulating the expression of pro-apoptotic proteins, in particular $B a x$. Although the precise mechanism by which other co-expressed factors down-regulate the expression of Bax still needs to be determined, the finding that WT1 suppresses $\mathrm{Bax}$ accumulation in GCs provides important insight into the regulation of apoptosis during early folliculogenesis.

The anti-apoptotic role of WT1 during urogenital development was first established by Kreidberg et al. [5]. To investigate the role of WT1 in GC apoptosis, serum was removed to induce spontaneous apoptosis [14]. Consistent with the role of WT1 in the regulation of ovarian FSH receptor expression in GCs [4], WT1 (-KTS) seems to play a significant role in enhancing cell survival, although both isoforms increased the proportion of viable cells under serum deprivation conditions (Figure 3A). In addition, the observations of decreased TdT-positive cells, marker of DNA fragmentation (Figure 4), are in agreement with previous reports on the anti-apoptotic action of WT1 in extragonadal cells [5]. Another important regulator of the apoptotic process is the caspase family of proteases [18]. Increased permeability of mitochondria under the influence of Bax results in the activation of caspases 3 and 7 as the final common pathway of apoptosis [24,30]. In addition to confirming Bax regulation by WT1 in GCs, we demonstrated anti-apoptotic effects of WT1 (-KTS). According to a previous report, WT1 (-KTS) induced apoptosis by upregulating caspase 3 in osteosarcoma cells [31]. Conversely, WT1 (-KTS) was found to inhibit apoptosis in GCs by down-regulating caspase 3 as strongly as FSH (Figure 3B). We also observed an anti-apoptotic effect of WT1 (+KTS), although it was not statistically significant. WT1 (+KTS) has been reported to inhibit apoptosis by down-regulating Bax in several human leukemic cells [11].
However, WT1 (+KTS) may affect GC survival via a different mechanism from WT1 (-KTS), as it played no role in the regulation of Bax expression (Figure 1).

To our knowledge, this is the first study demonstrating the role of WT1 in GC apoptosis and survival. Our observations corroborate previous reports on the antiapoptotic role of WT1 in non-gonadal cells [11]. We suggest that WT1 activation is necessary to maintain GC survival during the early stages of follicle development, and that WT1 can influence apoptosis at a number of points, including regulation of an upstream activator (Bax), and downstream effectors (caspases 3 and 7). Thus, WT1 may be a key regulator responsible for enhancing GC survival, with a fine-tuning role during the early stages, which take place in a relatively poor vascular environment similar to serum starvation. As more blood supply reaches the follicles and factors from the serum perfuse the cells, WT1 may counteract some proapoptotic factors until the cells can sufficiently respond to FSH. A better understanding of how apoptosis is regulated during the early FSH-independent follicular stages may improve our ability to manipulate fertility and the timing of the menopause.

\section{Conclusion}

These findings suggest that activation of WT1 is necessary for maintenance of GC survival during early stage of follicles and WT1 can play a role in protecting apoptosis through the regulation of upstream activator (Bax), as well as through regulation of downstream effecter (caspases 3 and 7). Collectively, our findings allow us to draw the conclusion that the fate of the follicles during their development may be determined by the changing pattern of WT1 and FSH receptor expression.

\section{Competing interests}

The authors declare that they have no competing interests.

\section{Authors contributions}

$J R$ participated in design, supervision of experiments, interpretation of data, revising and final approval of the article. MP, YC and HC participated in doing experiments, data analysis, literature review, and helped to draft the manuscript. All authors read and approved the final manuscript.

\section{Acknowledgments}

This work was supported by National Research Foundation of Korea Grant funded by the Korean Government [NRF-2014R1A1A2053601, and 2013R1A1A2057826].

Received: 3 July 2014 Accepted: 2 December 2014

Published online: 10 December 2014

\section{References}

1. Hirshfield AN: Development of follicles in the mammalian ovary. Int Rev Cytol 1991, 124:43 101

2. Chun SY, McGee EA, Hsu SY, Minami S, LaPolt PS, Yao HH, Bahr JM, Gougeon A, Schomberg DW, Hsueh AJ: Restricted expression of WT1 messenger ribonucleic acid in immature ovarian follicles, uniformity in mammalian and avian species and maintenance during reproductive senescence. Biol Reprod 1999, 60:365 373 
3. Hsu SY, Kubo M, Chun SY, Haluska FG, Housman DE, Hsueh AJ: Wilms tumor protein WT1 as an ovarian transcription factor, decreases in expression during follicle development and repression of inhibin-alpha gene promoter. Mol Endocrinol 1995, 9:1356 1366.

4. Yoon O, Roh J: Regulation of FSH receptor expression by the Wilms tumor 1 gene product (WT1) in immature rat granulosa cells. Mol Reprod Dev 2012, 79:368.

5. Kreidberg JA, Sariola H, Loring JM, Maeda M, Pelletier J, Housman D, Jaenisch R WT-1 is required for early kidney development. Cell 1993, 74:679 691.

6. Rao MK, Pham J, Imam JS, MacLean JA, Murali D, Furuta Y, Sinha-Hikim AP, Wilkinson MF: Tissue-specific RNAi reveals that WT1 expression in nurse cells controls germ cell survival and spermatogenesis. Genes Dev 2006, 20:147 152.

7. Williams GT, Smith CA: Molecular regulation of apoptosis, genetic controls on cell death. Cell 1993, 74:777 779.

8. Drummond IA, Rupprecht HD, Rohwer-Nutter P, Lopez-Guisa JM, Madden SL, Rauscher FJ 3rd, Sukhatme VP: DNA recognition by splicing variants of the Wilms tumor suppressor, WT1. Mol Cell Biol 1994, 14:3800 3809.

9. Wang ZY, Qiu QQ, Gurrieri M, Huang J, Deuel TF: WT1, the Wilms tumor suppressor gene product, represses transcription through an interactive nuclear protein. Oncogene 1995, 10:1243 1247.

10. Mayo MW, Wang CY, Drouin SS, Madrid LV, Marshall AF, Reed JC, Weissman BE, Baldwin AS: WT1 modulates apoptosis by transcriptionally upregulating the bcl-2 proto-oncogene. EMBO J 1999, 18:3990 4003.

11. Ito K, Oji Y, Tatsumi N, Shimizu S, Kanai Y, Nakazawa T, Asada M, Jomgeow T, Aoyagi S, Nakano Y, Tamaki H, Sakaguchi N, Shirakata T, Nishida S, Kawakami M, Tsuboi A, Oka Y, Tsujimoto Y, Sugiyama H: Antiapoptotic function of 17AA(+) WT1 (Wilms tumor gene) isoforms on the intrinsic apoptosis pathway. Oncogene 2006, 25:4217 4229.

12. Loeb DM: WT1 influences apoptosis through transcriptional regulation of BCl-2 family members. Cell Cycle 2006, 5:1249 1253.

13. Mosmann T: Rapid colorimetric assay for cellular growth and survival, application to proliferation and cytotoxicity assays. $J$ Immunol Methods 1983, 65:55 63.

14. Tilly JL, Billig H, Kowalski Kl, Hsueh AJ: Epidermal growth factor and basic fibroblast growth factor suppress the spontaneous onset of apoptosis in cultured rat ovarian granulosa cells and follicles by a tyrosine kinasedependent mechanism. Mol Endocrinol 1992, 6:1942 1950.

15. Harman SM, Louvet JP, Ross GT: Interaction of estrogen and gonadotrophins on follicular atresia. Endocrinology 1975, 96:1145 1152.

16. Hirshfield AN: Effect of a low dose of pregnant mares serum gonadotropin on follicular recruitment and atresia in cycling rats. Biol Reprod 1986, 35:113 118

17. Hughes FM Jr, Gorospe WC: Biochemical identification of apoptosis (programmed cell death) in granulosa cells, evidence for a potential mechanism underlying follicular atresia. Endocrinology 1991 129:2415 2422.

18. Markstrm E, Svensson EC, Shao R, Svanberg B, Billig H: Survival factors regulating ovarian apoptosis- dependence on follicle differentiation. Reproduction 2002, 123:23 30.

19. McGee EA, Hsueh AJ: Initial and cyclic recruitment of ovarian follicles. Endocr Rev 2000, 21:200 214

20. Asselin E, Xiao CW, Wang YF, Tsang BK: Mammalian follicular development and atresia, role of apoptosis. Biol Signals Recept 2000, 9:87 95.

21. Tilly JL, Kowalski KI, Johnson AL, Hsueh AJ: Involvement of apoptosis in ovarian follicular atresia and postovulatory regression. Endocrinology 1991, 129:2799 2801.

22. Roh J, Bae J, Lee K, Mayo K, Shea L, Woodruff TK: Regulation of Wilms tumor gene expression by nerve growth factor and follicle-stimulating hormone in the immature mouse ovary. Fertil Steril 2009, 91:1451 1454.

23. Antonsson B, Martinou JC: The BCl-2 protein family. Exp Cell Res 2000, 256:50 57.

24. Oltvai $\mathrm{ZN}$, Milliman $\mathrm{CL}$, Korsmeyer $\mathrm{SJ}$ : $\mathrm{BCl}-2$ heterodimerizes in vivo with a conserved homolog, Bax, that accelerates programmed cell death. Cell 1993, 74:609 619.

25. Graidista P, Nawakhanitworakula R, Saekooa J, Dechsukhumc C, Fujise K: Anti-apoptotic function of T-KTS+, T-KTS-, WT1+/+ and WT1+/- isoforms in breast cancer. Asian Biomed 2010, 4:711 720.

26. Rauscher FJ 3rd, Morris JF, Tournay OE, Cook DM, Curran T: Binding of the Wilms tumor locus zinc finger protein to the EGR-1 consensus sequence. Science 1990, 250:1259 1262
27. Wang ZY, Qiu QQ, Enger KT, Deuel TF: A second transcriptionally active DNA-binding site for the Wilms tumor gene product, WT1. Proc Natl Acad Sci U S A 1993, 90:8896 8900.

28. Hewitt SM, Hamada S, McDonnell TJ, Rauscher FJ 3rd, Saunders GF: Regulation of the proto-oncogenes $b c l-2$ and c-myc by the Wilms tumor suppressor gene WT1. Cancer Res 1995, 55:5386 5389

29. Tilly JL, Tilly Kl, Kenton ML, Johnson AL: Expression of members of the $b c l-2$ gene family in the immature rat ovary, equine chorionic gonadotropinmediated inhibition of granulosa cell apoptosis is associated with decreased Bax and constitutive bcl-2 and bcl-xlong messenger ribonucleic acid levels. Endocrinology 1995, 136:232 241.

30. Lakhani SA, Masud A, Kuida K, Porter GA Jr, Booth CJ, Mehal WZ, Inayat I, Flavell RA: Caspases 3 and 7, key mediators of mitochondrial events of apoptosis. Science 2006, 311:847 851

31. Morrison DJ, English MA, Licht JD: WT1 induces apoptosis through transcriptional regulation of the proapoptotic $\mathrm{BCl}-2$ family member Bak. Cancer Res 2005, 65:8174 8182

doi:10.1186/s13048-014-0118-y

Cite this article as: Park et al:: Wilms tumor suppressor gene (WT1) suppresses apoptosis by transcriptionally downregulating $B A X$ expression in immature rat granulosa cells. Journal of Ovarian Research 2014 7:118

\section{Submit your next manuscript to BioMed Central and take full advantage of:}

囚Convenient online submission

$\otimes$ Thorough peer review

$\otimes$ No space constraints or color $\nabla$ gure charges

QImmediate publication on acceptance

\Inclusion in PubMed, CAS, Scopus and Google Scholar

$\otimes$ Research which is freely available for redistribution 